\title{
Constitutively Photomorphogenic 1 Reduces the Sensitivity of Chronic Lymphocytic Leukemia Cells to Fludarabine Through Promotion of Ubiquitin-Mediated P53 Degradation
}

\author{
Chunling Fu ${ }^{a, b} \quad$ Xuanxuan Shi ${ }^{a}$ Yanqing Gong ${ }^{b}$ Yan Wan ${ }^{b}$ Zengtian Sun ${ }^{b}$ \\ Hengliang Shi $^{\mathrm{b}}$ Zhenzhen Wang $^{\mathrm{b}}$ Christian Marinaccio ${ }^{c}$ John D Crispino ${ }^{c}$ \\ Kailin $\mathrm{Xu}^{\mathrm{a}, \mathrm{b}}$
}

aBlood Diseases Institute, Xuzhou Medical University, Xuzhou, bDepartment of Hematology, The Affiliated Hospital of Xuzhou Medical University, Xuzhou, China, 'Division of Hematology/Oncology, Northwestern University, Chicago, USA

\section{Key Words}

$\mathrm{Cll} \cdot \mathrm{COP} 1 \cdot$ Ubiquitin-dependent degradation $\bullet \mathrm{F}+\mathrm{C}$ therapy $\bullet$ Apoptosis

\begin{abstract}
Background/Aims: Chronic Lymphocytic leukemia (CLL) is characterized by accumulation of cells in the G0/G1 phase of the cell cycle and resistance to apoptosis due to gene mutation or abnormal gene expression. In our previous study, constitutively photomorphogenic 1 (COP1) was shown to be upregulated in Binet C-phase CLL patients. Based on the negative regulation of COP1 in the repair of DNA damage, we further studied the function of COP1 in CLL cell apoptosis induced by fludarabine in vitro and in vivo. Methods: We analyzed the sensitivity of primary CLL cells to the fludarabine by CCK-8, and detected the expression of p53 in cells after drug treatment by western blot. Next, we constructed COP1 overexrpessing CLL cell line HG3, and analyzed the effect of COP1 overexpression on the HG3 cell's apoptosis, and HG3 transplant mice survival with drug treatment. Results: Here, we found that primary CLL cells with high expression of COP1 showed low sensitivity to the drug and presented delayed enrichment of p53 protein than cells with low COP1 expressed. COP1 overexpression reduced HG3 cell sensitivity to the fludarabine treatment and inhibited cell apoptosis, and also retarded itself via autoubiquitination. The further study showed that COP1 promoted ubiquitindependent p53 degradation, which further disrupts the formation of the p53-Brn-3a complex and activation of $\mathrm{Bcl}-2$ transcription. Moreover, mice engrafted with cells overexpressing COP1 showed a shortened survival, increased tumor cells burden in spleen and bone marrow
\end{abstract}

C. Fu and X. Shi contributed equally to the work. 


\section{Cellular Physiology Cell Physiol Biochem 2018;50:2314-2328

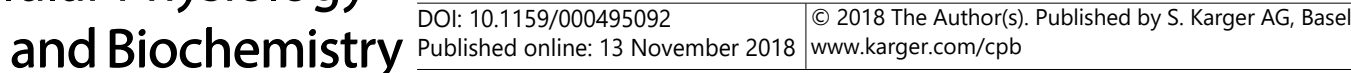 \\ Fu et al.: Constitutively Photomorphogenic 1 Reduces the Sensitivity of Chronic \\ Lymphocytic Leukemia Cells to Fludarabine}

$(B M)$, and reduced tumor cell apoptosis even when fludarabine combined cyclophosphamide $(\mathrm{F}+\mathrm{C})$ therapy was administered. Conclusion: This study demonstrates that COP1 contributes to drug resistance of CLL cells to the fludarabine treatment in vitro and in vivo.

\section{Introduction}

Chronic lymphocytic leukemia (CLL) is the most common form of B cell leukemia in adults and is particularly prevalent in Western countries [1]. The incidence of this disease is gradually increasing in China [2]. Gene mutation or abnormal expression is common in CLL, such as p53 inactivated in 10-15\% of CLL patients [3-5], and Bcl-2 is upregulated, leading to an elevated $\mathrm{Bcl}-2 / \mathrm{Bax}$ ratio which is a major factor causing accumulation of cells in the G0/ G1 phase of the cell cycle and resistance to apoptosis [6, 7]. In a previous study we showed that COP1 was upregulated in Binet C-phase CLL patients and positively correlated with ZAP-70 expression [8]. This result suggests that COP1 function may be important in CLL development.

COP1 is first defined as a central regulator of photomorphogenic development in plants [9], and subsequently in mammals [10]. It consists of 3 functional domains: a RING-finger domain, a coiled-coil domain, and a WD40 domain which are highly conserved from plants to mammals [11]. COP1 is characterized by E3 ubiquitin ligase activity, which targets substrate for proteasome-dependent degradation and plays important roles in many biological responses in mammals [12]. P53 is a substrate of COP1, and directly interacts with the nonphosphorylated form of COP1 in the nucleus [13]. COP1 promotes 553 proteasome-mediated degradation in a ubiquitin-dependent manner and inhibits p53-dependent transcription and apoptosis. In response to DNA damage, ATM phosphorylates COP1 on Ser387 and stimulates a rapid auto-degradation mechanism $[14,15]$. ATM induces the translocation of COP1 from the nucleus to the cytoplasm, and disrupts the COP1-p53 complex resulting in abrogation of ubiquitination and degradation of p53 [14]. P53 is directly involved in cell cycle by regulating p21 levels, and, as a transcriptional factor, it complexes with Brn-3a at the Bcl-2 promoter site to control Bcl-2 expression, which regulates apoptosis $[16,17]$.

COP1 is frequently overexpressed in breast, ovarian, hepatic and gastric cancer where it promotes tumorigenicity via inhibition of p53 activity [18-20]. COP1 targets CCAAT/ enhancer-binding protein $(\mathrm{C} / \mathrm{EBPa})$ for degradation and induces acute myeloid leukemia via Trib1 [21]. COP1 also constitutively regulates c-Jun protein stability and functions as a tumor suppressor in mice. COP1 hypomorphic mice spontaneously develop malignancies at a high frequency in the first year of life and are highly susceptible to radiation-induced lymphomagenesis [22]. Although COP1 is involved in many biological processes in solid tumor development, its role in diseases of the hematopoietic system is less known, especially in chronic lymphocytic leukemia.

In the present study, we address the mechanism of COP1-mediated CLL cell resistance to fludarabine in vitro and in vivo. We show that CLL cells overexpressing COP1 have reduced sensitivity to fludarabine, and are less susceptible to apoptosis due to increased p53 degradation and disrupted formation of the p53-Brn-3a complex, which promotes Bcl2 transcription and expression. Moreover, mice transplanted with CLL cells overexpressing COP1 and treated with $\mathrm{F}+\mathrm{C}$ show higher tumor burden, less apoptosis in spleen and BM, and shortened survival when compared to mice with CLL cells overexpressing GFP. These results suggest that COP1 is a negative regulator in CLL apoptosis under a fludarabine treatment regimen. 


\section{Cellular Physiology Cell Physiol Biochem 2018;50:2314-2328

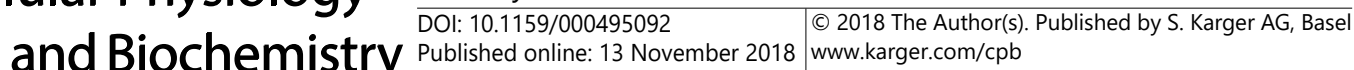

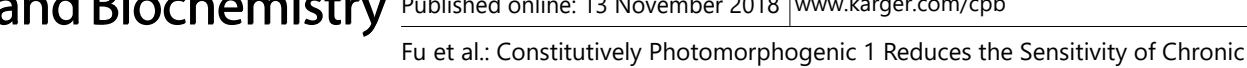

Lymphocytic Leukemia Cells to Fludarabine

\section{Materials and Methods}

\section{Cell line and reagents}

CLL cell line HG3, a kind gift by Anders Rosén from Linköping University, was cultured in RPIM1640 medium supplied with $10 \%(\mathrm{v} / \mathrm{v}) \mathrm{FBS}$ at $37{ }^{\circ} \mathrm{C}$ in $5 \% \mathrm{CO}_{2}$ incubator [23]. The stable cell lines HG3GFP and HG3-COP1 were derived with pWXLD-GFP and pWXLD-GFP-COP1 lentivirus infection, and then selected with puromycin. The stable cell line HG3-m-GFP and HG3-m-COP1 were obtained by two rounds of lentiviral infection with two different constructs. HG3 cells were first infected with mCherry-Luciferase fusion lentivirus to obtain the HG3-mCherry stable line, and then infected a second time with pWXLD-GFP and pWXLD-GFP-COP1 lentivirus, to derive the HG3-m-GFP and HG3-m-COP1 cell lines.

COP1 antibody was purchased from Bethyl Laboratories. Inc (Montgomery, AL, USA). Brn-3a was purchased from Santa Cruz (Santa Cruz, CA, USA), cleaved-caspase 3 was purchased from Abcam (Cambridge, MA, UK), p53 and Ub antibodies were purchased from Cell Signaling Technology (Danvers, MA, USA), $\beta$-actin, flag antibody, Goat anti-Rabbit IgG, Rabbit anti-Mouse IgG and MG-132 were obtained from Sigma (SigmaAldrich, St. Louis, MO, USA). Fludarabine and Cyclophosphamide were purchased from Cayman (Michigan, USA). CCK-8 and Annexin V/7-AAD kit was from ebioscience (San Diego, CA, USA)). D-firefly luciferin (St. Louis, MO, USA). Stemspan medium and cytokine were purchased from stem cell (Stem Cell Technologies, Vancouver, BC, Canada). CCK-8 kit was purchased from Ruibo (Guangdong, China).

\section{Patient sample}

CLL samples were obtained from the Affiliated Hospital of Xuzhou Medical College according to the diagnostic criteria for CLL between August 2013 and July 2017. PBMCs were isolated from heparinized blood obtained from 6 CLL patients, and total cells were cultruled in stemspan medium containing 10ng/ $\mathrm{ml}$ of hSCF, hIL-3 and hIL-11 with fludarabine. The cells were collected for CCK-8 detection or western blot.

\section{Animals}

Female 6-8 week-old NPG (NOD-Prkdc scid Il2rg ${ }^{\text {null }}$ ) mice lacking B,T and NK cells were obtained from Vital River Laboratories Co., Ltd. (Beijing, China). The NPG mice were inoculated with $1 \times 10^{5} \mathrm{HG} 3-\mathrm{m}$-GFP or HG3-m-COP1 cells by tail injection to establish the CLL mouse model. All experimental procedures were performed in accordance with the guidelines for laboratory animals established by the Xuzhou Medical College Animal Care and Use Committee.

\section{Cell proliferation and apoptosis assay}

The stable cell lines HG3-GFP and HG3-COP1 were grown in 96-well plates. 2000 cells per well were treated with different doses of fludarabine for $72 \mathrm{~h}$. The cells were then incubated for $4 \mathrm{~h}$ with CCK-8, and the absorbance at $450 \mathrm{~nm}$ was determined with a microplate reader (Wellscan MK3, Labsystems Dragon, Helsinki, Finland), and the EC50 was calculated using Graphpad Prism 6.

To measure apoptosis, cells were incubated with fludarabine for 0,6 or $12 \mathrm{~h}$ and then stained with Annexin V/7-AAD apoptosis kit according the manual. The degree of apoptosis was quantified by flow cytometry.

\section{Co-immunoprecipitation and immunoblotting}

Protein lysates were diluted to $1 \mathrm{mg} / \mathrm{ml}$, and incubated with primary antibody against p53 overnight at $4{ }^{\circ}$. The next day protein $\mathrm{A} / \mathrm{G}$-agarose suspension was added to the sample and incubated for at least 3 hours at $4 \stackrel{\circ}{\circ} \mathrm{C}$ on a rocking platform. The agarose-antibody-antigen complex was centrifuged $(20 \mathrm{~s}$ at 12,000 $\times \mathrm{g}$ ) and supernatant discarded. The beads were washed once and elution was performed with $2 \times$ loading buffer boiled for 3 minutes. The immunoprecipitated protein was then run on SDS-PAGE gel and transferred to a $0.45 \mu \mathrm{m}$ pore size PVDF membrane (Millipore, Billerica, MA, USA). After blocking with $5 \%$ non-fat milk, the membrane was probed with primary antibodies at $4^{\circ} \mathrm{C}$ overnight and secondary antibodies at room temperature for $1 \mathrm{~h}$. Bound antibodies were detected by the Pierce ECL Plus Western Blotting Substrate (Thermo Fisher Scientific) and visualized by ImageQuant LAS 4000 (GE, Fairfield, CT, USA). 


\section{Cellular Physiology Cell Physiol Biochem 2018;50:2314-2328

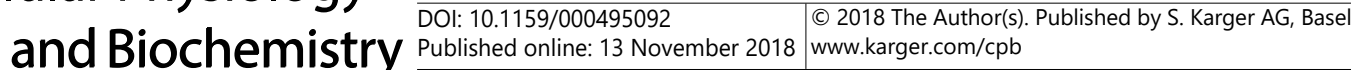 \\ Fu et al.: Constitutively Photomorphogenic 1 Reduces the Sensitivity of Chronic \\ Lymphocytic Leukemia Cells to Fludarabine}

\section{Dual luciferase reporter assay}

The P2 promoter sequence of Bcl-2 was compounded from Shenggong Company (Shanghai, China), and then inserted into firefly luciferase PGL3-Basic plasmid (Promega Corporation, Madison, WI, USA) between the BgIII and HindIII enzyme site by us. To perform the luciferase assay, COS7 cells were grown in a 12-well plate, and transduced with Renilla luciferase pRL-TK and PGL3-Basic-Bcl-2-P2, plus the empty flag vector, flag-tagged Cop1 and flag-Brn-3a plasmid. After 24 hours, cells were treated with fludarabine for 10 h. Half of the cell sample was used for western blotting, and the other half was used for a luciferase assay according to the manufacturer's protocol. The assay was performed in triplicate. Renilla luciferase activity was normalized to firefly luciferase activity for each well to normalize for cell number and transfection efficiency.

\section{In vivo imaging}

For in vivo imaging, a NightOWLIILB 983 small-animal in vivo imaging system (Berthold Technologies, Germany) was used. NPG mice were anesthetized with a 3:1 mixture of ketamine and xylazine intraperitoneally. After 5-10 min of anesthetic induction, an aqueous solution of the substrate, D-firefly luciferin, was injected into the peritoneal cavity $(15 \mathrm{mg} / \mathrm{ml}$ at a dose of $150 \mathrm{mg} / \mathrm{kg}$ body weight). After 15 $\mathrm{min}$, the animals were immediately placed in a chamber and imaged. We calculated the average radiance using this formula: average radiance $=$ photons $/ \mathrm{sec} / \mathrm{cm}^{2} / \mathrm{sr}$, which represents the number of photons per second that leave a square centimeter of tissue and radiate into a solid angle of one steradian (sr). Measurements in units of radiance automatically take into account camera settings and allow comparing data obtained from different animals or from the same animal in different days.

\section{Histopathology and immunohistochemistry}

Spleen and BM were isolated, fixed in 10\% formalin for $72 \mathrm{~h}$, decalcified and paraffin embedded. Sections were cut at $4 \mu \mathrm{m}$ thickness, placed on slides and processed for hematoxylin and eosin staining. For immunohistochemistry, the slides were in xylene for dewaxing and then histological grade ethanol for rehydration, and in $1 \times$ citrate unmasking solution for antigen retrieval. Endogenous peroxidases were blocked with $3 \% \mathrm{H}_{2} \mathrm{O}_{2}$ for $10 \mathrm{~min}$. A protein block step was performed with $5 \% \mathrm{BSA}$ for $2 \mathrm{~h}$. Tissues were incubated overnight at $4^{\circ} \mathrm{C}$ with primary rabbit anti cleaved-caspase 3 antibody and with a polink-2 plus polymer HRP detection system according to the manufacturer's instructions.

\section{Statistical analysis}

The statistical analyses were performed using GraphPad Prism 6 software. Student's $t$ test and ANOVA were used to compare the difference in values between groups. Survival was analyzed with Kaplan-Meier curves, and significance estimated with a log-rank test. $\mathrm{P}<0.05$ was considered as significant difference.

\section{Results}

CLL cells with COP1 overexpression are less sensitive to fludarabine and presented decreased cell apoptosis ratio

To better understand the how the high level of COP1 in CLL patients influences CLL cell survival and apoptosis, we first treated primary mononuclear cells from 3 pairs of CLL patients with fludarabine. We found that primary CLL cells with high expression of COP1 showed significantly low sensitivity to the drug at $8 \mathrm{~h}\left({ }^{*} \mathrm{p}=0.032\right)$ compared with cells with low COP1 expressed (Fig. 1A). Of note, high expression of COP1 presented delayed enrichment of p53 protein in CLL primary cells which were treated with fludarabine for $4 \mathrm{~h}$ (Fig. 1B). Next, we constructed HG3 cell line with lentiviruses expressing either GFP alone or GFP and COP1, treated these cells with increasing doses of fludarabine, and measured cell viability at $72 \mathrm{~h}$. We found that COP1 overexpression reduced HG3 sensitivity to fludarabine (Fig. 1C) with IC50 values of $4.8 \mu \mathrm{M}$ for HG3-COP1 and $2.7 \mu \mathrm{M}$ for HG3-GFP cells. Moreover, COP1 overexpression decreased the extent of apoptosis of HG3 cells: when the same dose of fludarabine treatment was administered, the HG3-COP1 cell apoptosis rate were $7.8 \%$ and $8.2 \%$ versus $11.8 \%$ and $16.5 \%$ for the HG3-GFP cells at $6 \mathrm{~h}$ and $12 \mathrm{~h}$, as determined by 


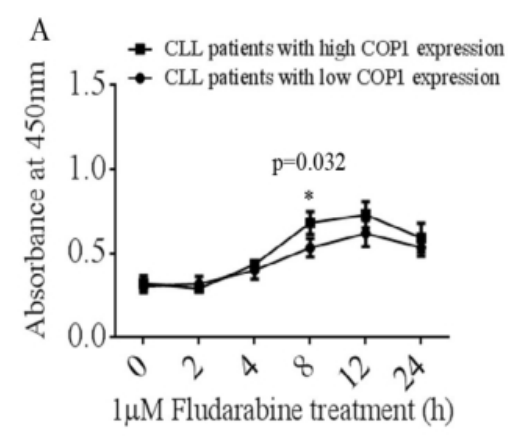

B
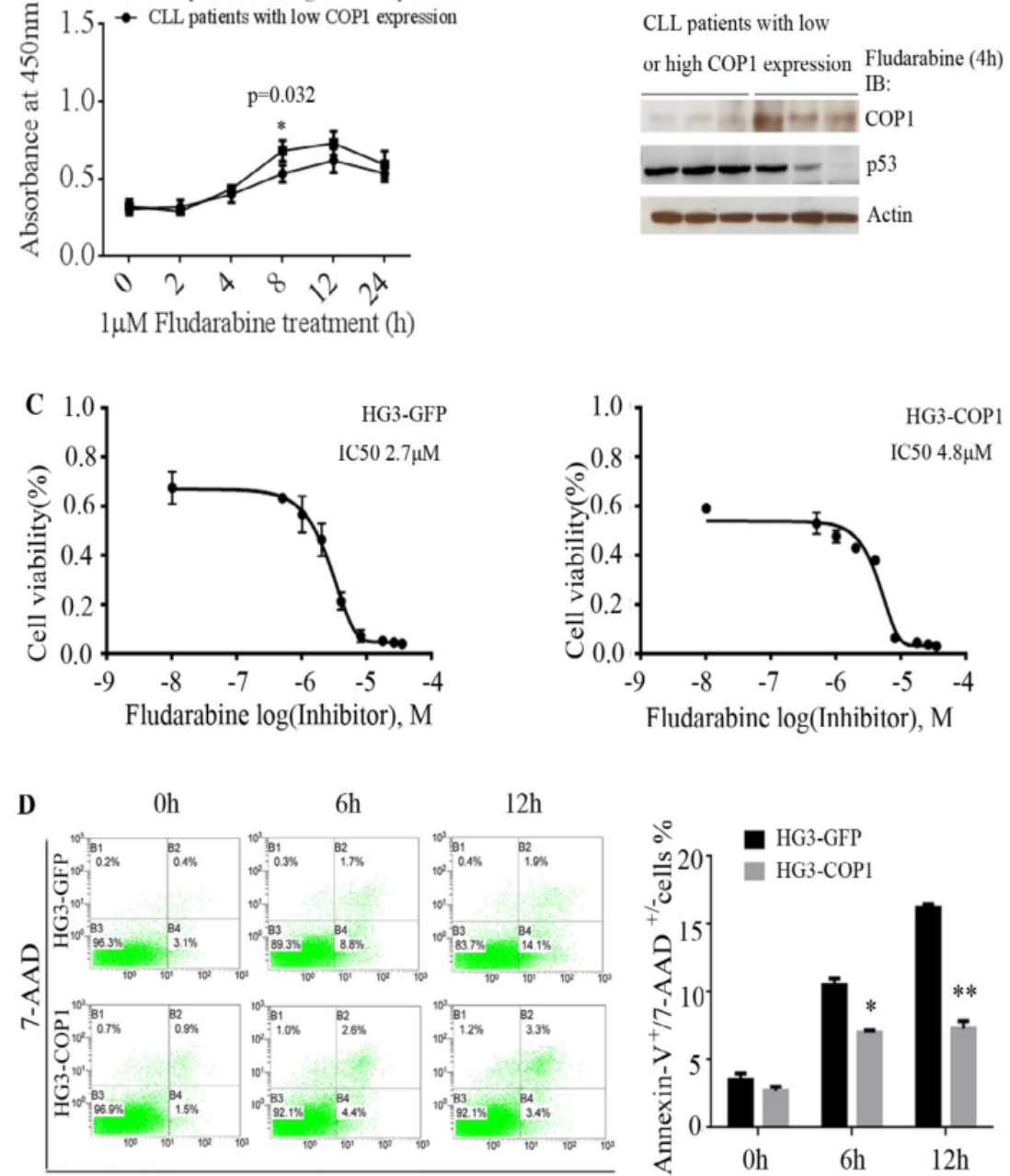

Annxin-V-APC (Fludarabine)
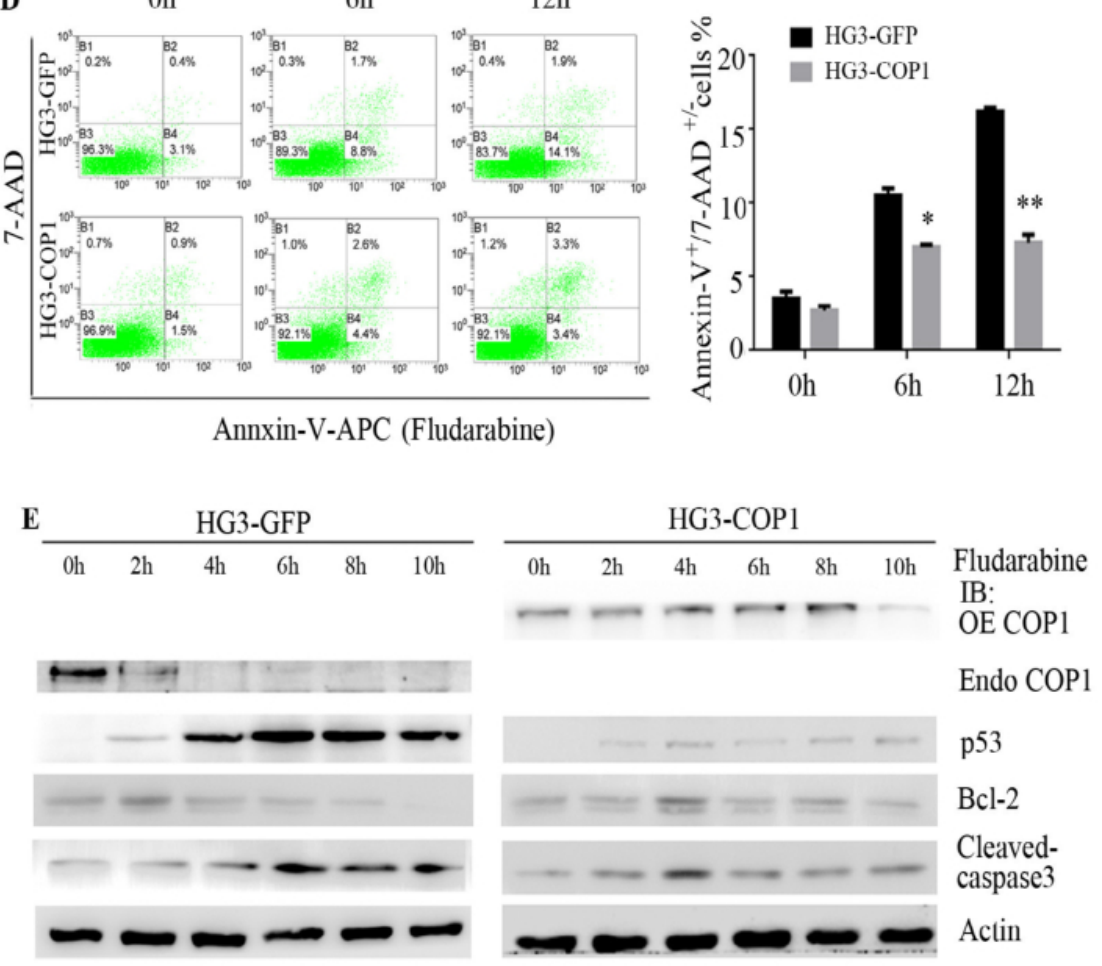

Fig. 1. COP1 confers resistance to fludarabine. (A and B) primary CLL cells' proliferation was analyzed by CCK-8, and p53 expression was detected by WB. (C) Inhibition curves of HG3-GFP and HG3-COP1 cells treated with different doses of fludarabine for 72 hours. (D) 7-AAD and Annexin V staining for determining apoptosis of HG3-GFP and HG3-COP1 cells treated with fludarabine for 0, 6 and 12 hours. Quantification of three independent experiments are shown $(\mathrm{p}<0.05, \mathrm{p}<0.01)$. (E) Protein expression of COP1, p53, Bcl-2 and cleaved-caspase 3 in HG3-GFP and HG3-COP1 cells at different time points. Actin is shown as a loading control.

\section{KARGER}




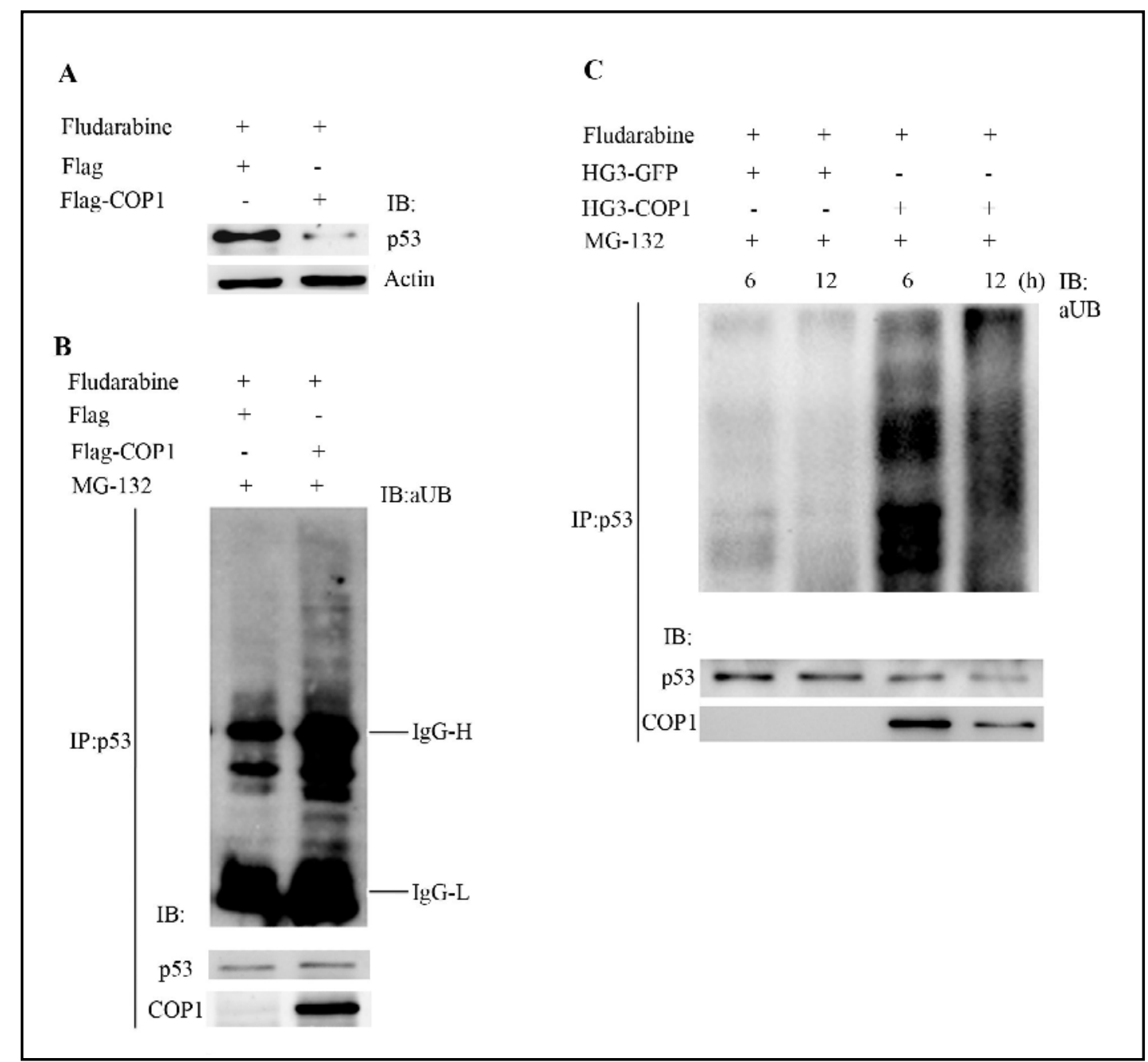

Fig. 2. COP1 promoted p53 degradation by ubiquitin-mediated in HG3 cells. (A) Immunoblot of p53 in COS7 cells overexpressing empty vector or flag-COP1 after 12 hours of fludarabine treatment. Actin is shown as a loading control. (B) Immunoblot of ubiquitin following immunoprecipitation of p53 in COS7 cells overexpressing empty vector or flag-COP1 after 12 hours of fludarabine treatment and inhibition of proteasome through MG-132. Non-specific signal from heavy and light IgG chains is shown. (C) Immunoblot of ubiquitin following immunoprecipitation of p53 in HG3-GFP or HG3-COP1 cells after 6 or 12 hours of fludarabine treatment and inhibition of proteasome through MG-132.

Annexin V/7-AAD staining ( ${ }^{*} \mathrm{P}<0.05,{ }^{* *} \mathrm{P}<0.01$ ) (Fig. 1D). Next, apoptosis-related protein expression in the two cell lines was analyzed by western blot, we found that $\mathrm{p} 53$ and cleavedcaspase 3 levels were significantly increased while Bcl-2 and endogenous COP1 progressively decreased in HG3-GFP cells treated with fludarabine. Interestingly, HG3-COP1 cells didn't show COP1's degradation until 10 hours after drug treatment, and showed only a small increase of p53 as well as cleaved-caspase 3 levels. Moreover, there was almost no change in Bcl-2 protein level (Fig. 1E). Together, these results suggest that COP1 overexpression reduced HG3 apoptosis and sensitivity to fludarabine. 


\section{Cellular Physiology Cell Physiol Biochem 2018;50:2314-2328 \\ \begin{tabular}{l|l|l} 
and Biochemistry Published online: 13 November 2018 & $\begin{array}{l}\text { C } 2018 \text { The Author(s). Published by S. Karger AG, Basel } \\
\text { www.karger.com/cpb }\end{array}$
\end{tabular}}

Fu et al.: Constitutively Photomorphogenic 1 Reduces the Sensitivity of Chronic

Lymphocytic Leukemia Cells to Fludarabine

COP1 overexpression inhibits p53 expression induced by fludarabine and promotes ubiquitin-mediated p53 degradation in HG3 cells

To explain how COP1 increases HG3 resistance to fludarabine, we assayed changes of p53 level, a substrate of COP1 E3 ligase, in COS7 and HG3 cells treated with fludarabine. We found that p53 levels were significantly increased in COS7 cells after fludarabine treatment without exogenous COP1, whereas p53 was inhibited in COP1 overexpressing cells (Fig. 2A). To determine whether COP1 overexpression promoted ubiquitin-mediated p53 degradation, we treated COS7 cells with fludarabine and the proteasome inhibitor MG-132 to allow accumulation of ubiquitinated substrates. Transfection of COP1 led to a strong accumulation of ubiquitin products of p53 when compared to control cells (Fig. 2B).

We also examined the ubiquitin products of p53 in HG3 cells with or without COP1 overexpression. Treatment of HG3-GFP and HG3-COP1 cells with both MG-132 and fludarabine revealed that HG3-COP1 cells have strong accumulation of ubiquitinated p53 at $6 \mathrm{~h}$ and $12 \mathrm{~h}$ compared to HG3-GFP cells (Fig. 2C). These data suggest that COP1 increased ubiquitinated p53 level and accelerated ubiquitin-mediated p53 degradation in HG3 cells.

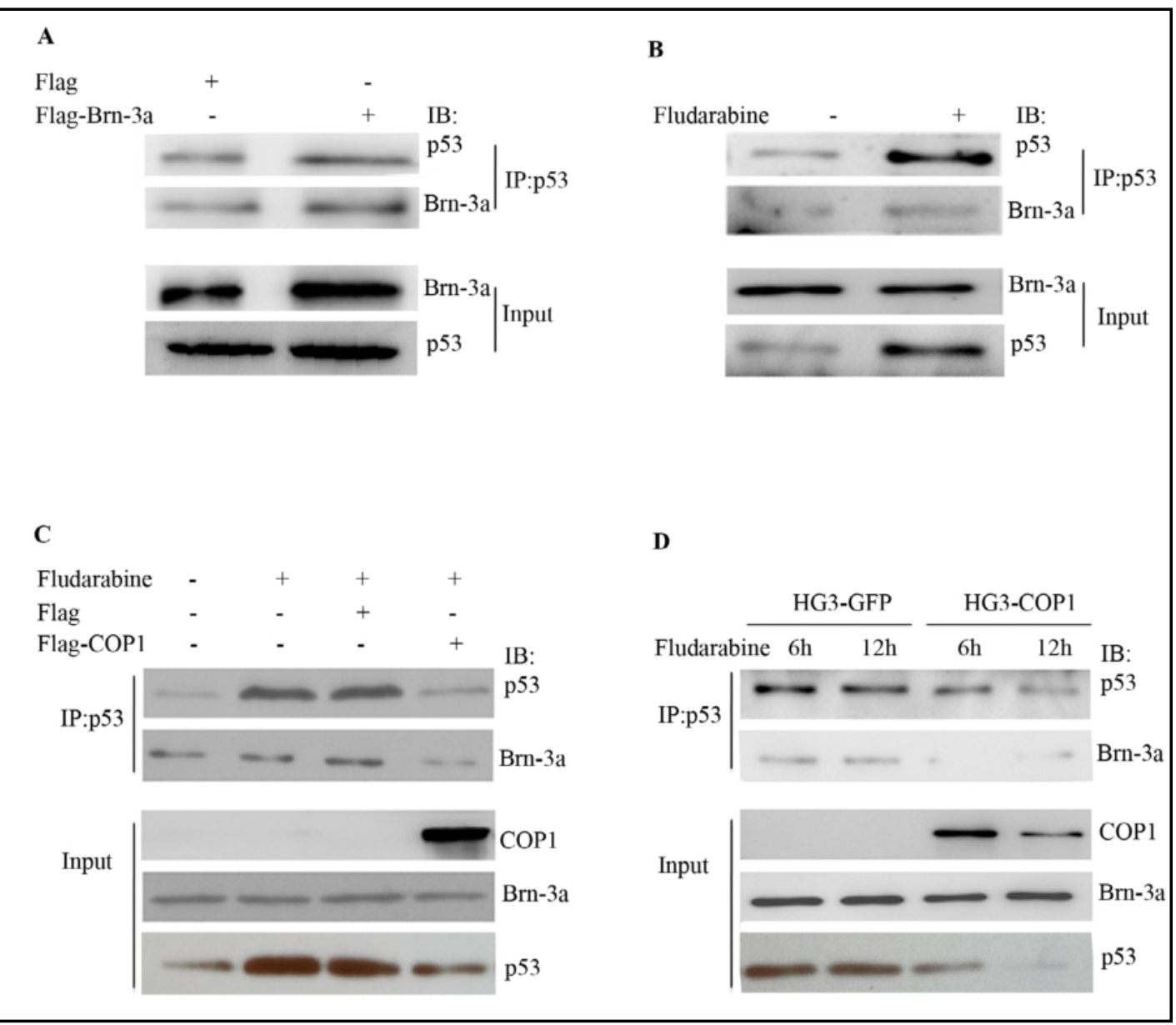

Fig. 3. Overexpression of COP1 disrupts the p53-Brn-3a interaction. (A) Co-immunoprecipitation of Brn3a and p53. COS7 cells were transfected with flag empty vector or flag-Brn3a. p53 immunoprecipitates were probed for p53 or Brn3a. (B) Co-immunoprecipitation of Brn-3a and p53 in HG3 cells untreated or treated for 12 hours with fludarabine. (C) Co-immunoprecipitation of Brn-3a and p53 in untransfected COS7 cells or COS7 cells transfected with flag-COP1 with or without fludarabine treatment for 12 hours. (D) Coimmunoprecipitation of Brn-3a and p53 in HG3-GFP or HG3-COP1 after fludarabine treatment for 6 or 12 hours. 
Lymphocytic Leukemia Cells to Fludarabine

COP1 overexpression disrupts the p53-Brn-3a complex

In order to analyze how COP1 overexpression influences Bcl-2 and cleaved-caspase 3 expression through p53, we focused on the formation of p53-Brn-3a complex because it can regulate Bcl-2 promoter. In parental COS7 and HG3 cells, there is an interaction between p53 and Brn-3a, and this interaction was increased by exogenous Brn-3a transduction in COS7 cells. A similar increase of the interaction through p53 upregulation was observed in HG3 cells treated with fludarabine (Fig. 3A and B). However, the p53-Brn-3a interaction was disrupted in COP1 overexpressing COS7 cells in the presence of fludarabine compared to control COS7 cells transduced with empty vector (Fig. 3C). A similar diminution of the p53-Brn-3a interaction was observed in COP1 overexpressing HG3 cells in the presence of fludarabine compared to H3G-GFP control cells (Fig. 3D).

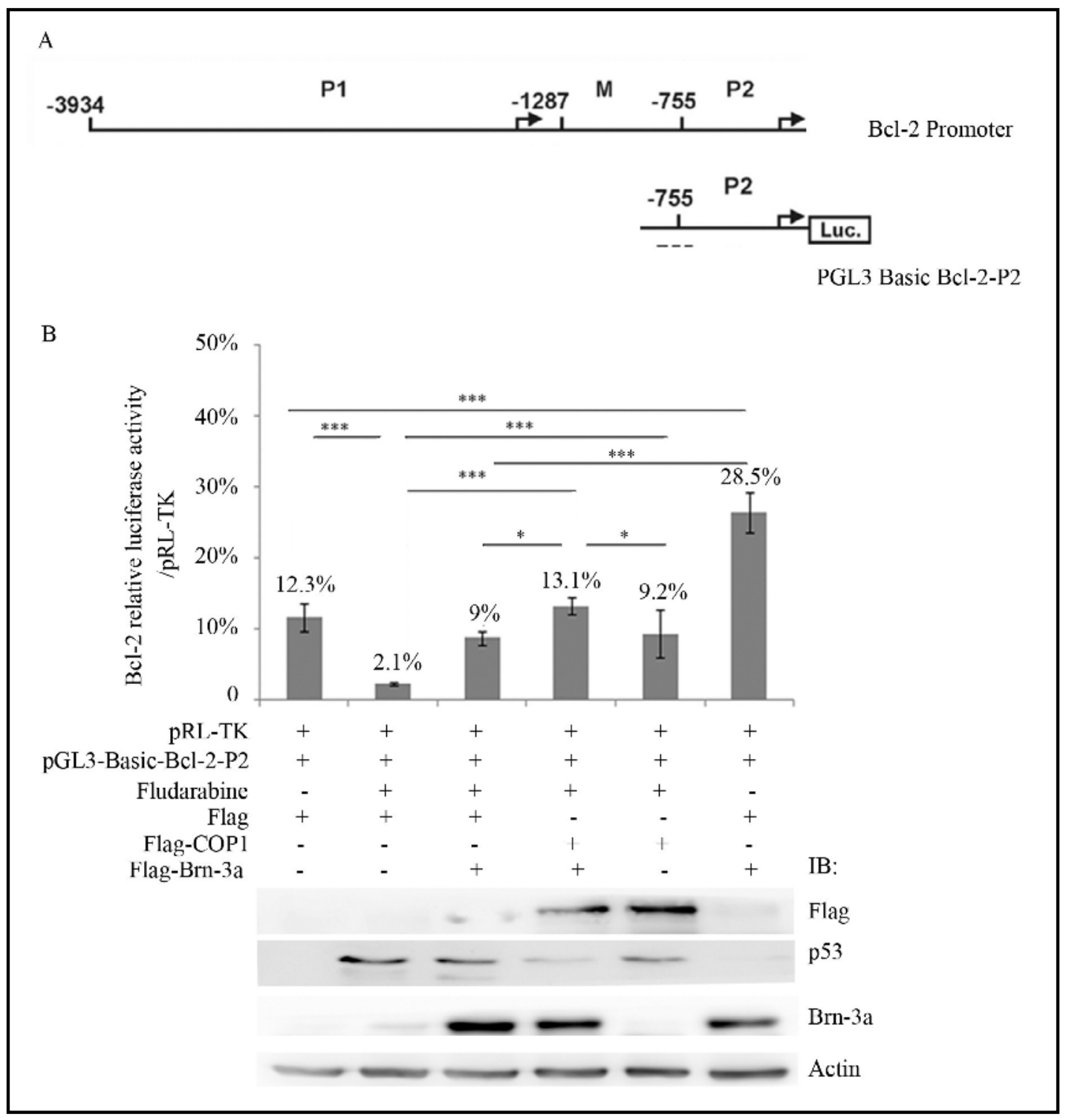

Fig. 4. Overexpression of COP1 increases Bcl-2 promoter activity. (A) The P2 promoter of Bcl-2 (about 755 bp) was cloned into PGL3 Basic plasmid. (B) COS7 cells were co-transfected with Renilla luciferase pRLTK, Bcl-2-P2 plasmid. After 4 hours, the resulting cells were co-transfected with either flag empty vector or flag-COP1 and with or without Brn-3a. After 24 hours, the cells were either treated or not treated with fludarabine for 12 hours and cell lysates prepared for a dual luciferase assay or immunoblotted for the flag epitope, p53, or Brn-3a. Actin is shown as a loading control. 


\section{Cellular Physiology Cell Physiol Biochem 2018;50:2314-2328 \begin{tabular}{ll|l} 
DOl: 10.1159/000495092 & (0) 2018 The Author(s). Published by S. Karger AG, Basel \\
and Biochemistry. & Published online: 13 November 2018 www.karger.com/cpb
\end{tabular} \\ Fu et al.: Constitutively Photomorphogenic 1 Reduces the Sensitivity of Chronic \\ Lymphocytic Leukemia Cells to Fludarabine}

COP1 overexpression enhanced the activation of the Bcl-2 promoter regulated by $\mathrm{p} 53-\mathrm{Brn}$ 3a complex

The balance of p53 and Brn-3a in the free and complexed forms is important for Bcl2 transcription $[17,24]$. To monitor the transcriptional regulation by these factors in the presence of COP1, we cloned the P2 promoter of Bcl-2 into a PGL3-Basic plasmid. Luciferase is expressed from this plasmid when the P2 promoter is activated with a baseline Bcl2 luciferase activity of $12.3 \%$ (Fig. 4A). The fludarabine treated group showed a lower activity $\left(2.1 \%,{ }^{* * *} \mathrm{P}<0.001\right)$ because p53 accumulation inhibits $\mathrm{Bcl}-2$ transcription. When Brn-3a was overexpressed in absence of fludarabine, luciferase activity was significantly increased $\left.\left(28.5 \%,{ }^{* * *} \mathrm{P}<0.001\right)\right)$, indicating transcriptional activation. Conversely, Brn-3a overexpression in presence of fludarabine treatment caused a reduction of luciferase activity near baseline levels $\left(9 \%,{ }^{* * *} \mathrm{P}<0.001\right)$ due to increased interaction between p53 and Brn3a.

Whereas fludarabine treatment alone resulted in p53 accumulation and low Bcl-2 transcriptional activity (2.1\%), COP1 overexpression alone in presence of fludarabine increased $\mathrm{Bcl}-2$ transcriptional activity to $9.2 \%,\left({ }^{* * *} \mathrm{P}<0.001\right)$ due to p53 degradation. Coexpression of COP1 and Brn-3a caused a marked decrease of p53 which further enhanced Bcl-2 transcriptional activity $\left(13.1 \%,{ }^{* * *} \mathrm{P}<0.001\right)$ due to high levels of Brn-3a. The Bcl-2 transcriptional activity from co-expression of COP1 and Brn-3a was higher than COP1 or Brn3 a alone overexpression $\left({ }^{*} \mathrm{P}<0.05\right)$. Together these results showed that COP1 regulates Bcl2 promoter activity by directly controlling the level of $\mathrm{p} 53$, and also disrupts the interaction between p53 and Brn-3a in the process of fludarabine-induced apoptosis (Fig. 4B).

The HG3-COP1 cell line transplant mice were low sensitivity to the F+C therapy

To investigate whether COP1 overexpression confers resistance to chemotherapy in vivo, we established CLL xenograft mouse model by injecting $1 \times 10^{5} \mathrm{HG} 3-\mathrm{m}-\mathrm{GFP}$ or HG3-mCOP1 cells co-expressing GFP, mCherry and luciferase into mice. Two-weeks after injection, we observed successful tumor cell engraftment by in vivo imaging (Fig. 5A). After confirming tumor engraftment, we treated mice with fludarabine in combination with cyclophosphamide daily for two weeks. After one week of therapy (day 21 after CLL cell inoculation), HG3-mCOP1 recipient mice showed very strong photon density by in vivo imaging (Fig. 5A). After two weeks of therapy (day 28 after CLL cell inoculation), all mice in the HG3-m-COP1 group died while one mouse from the HG3-m-GFP was still alive with a low photon density (Fig. 5A and B). Platelet and RBC counts decreased in the HG3-m-COP1 mice before they died (data not shown). Taken together, HG3-m-COP1 engrafted mice showed a shorter survival and decreased body weight when compared with HG3-m-GFP transplanted mice $(* * * \mathrm{P}<0.001)$ (Fig. 5C and D). Moreover, HG3-m-COP1 mice showed an increase in spleen weight with a mean value of $0.68 \mathrm{~g}$ versus a mean value of $0.47 \mathrm{~g}$ in $\mathrm{HG} 3-\mathrm{m}-\mathrm{GFP}$ control mice $\left({ }^{*} \mathrm{P}<0.05\right)$ (Fig. 5E and F).

COP1 overexpression promoted HG3 cells engraftment in spleen and BM

Histology of HG3-m-COP1 transplant mice revealed abnormal spleen and BM structure. Abundant clusters of small, intensely stained B lymphoid cells were seen in spleen and BM of HG3-m-COP1 mice when compared with HG3-m-GFP mice (Fig. 6A). Tumor cell burden of HG3-m-COP1 mice in the spleen was 280 clusters per section, significantly higher then HG3m-GFP mice with 120 CLL clusters $\left({ }^{* *} \mathrm{P}<0.01\right)$. Similarly, more CLL clusters were observed in BM in HG3-m-COP1 mice (Fig. 6B). Also, the total cells were isolated from spleen and BM after red cell lysis, the occupation of CLL cells was detected by flow cytometry. As the figure 7C showed that the CLL cells percentage was $\sim 55 \%$ and $\sim 22 \%$ in spleen or BM in HG3-mCOP1 mice compared with $\sim 46 \%$ and $\sim 18 \%$ in HG3-m-GFP mice. 


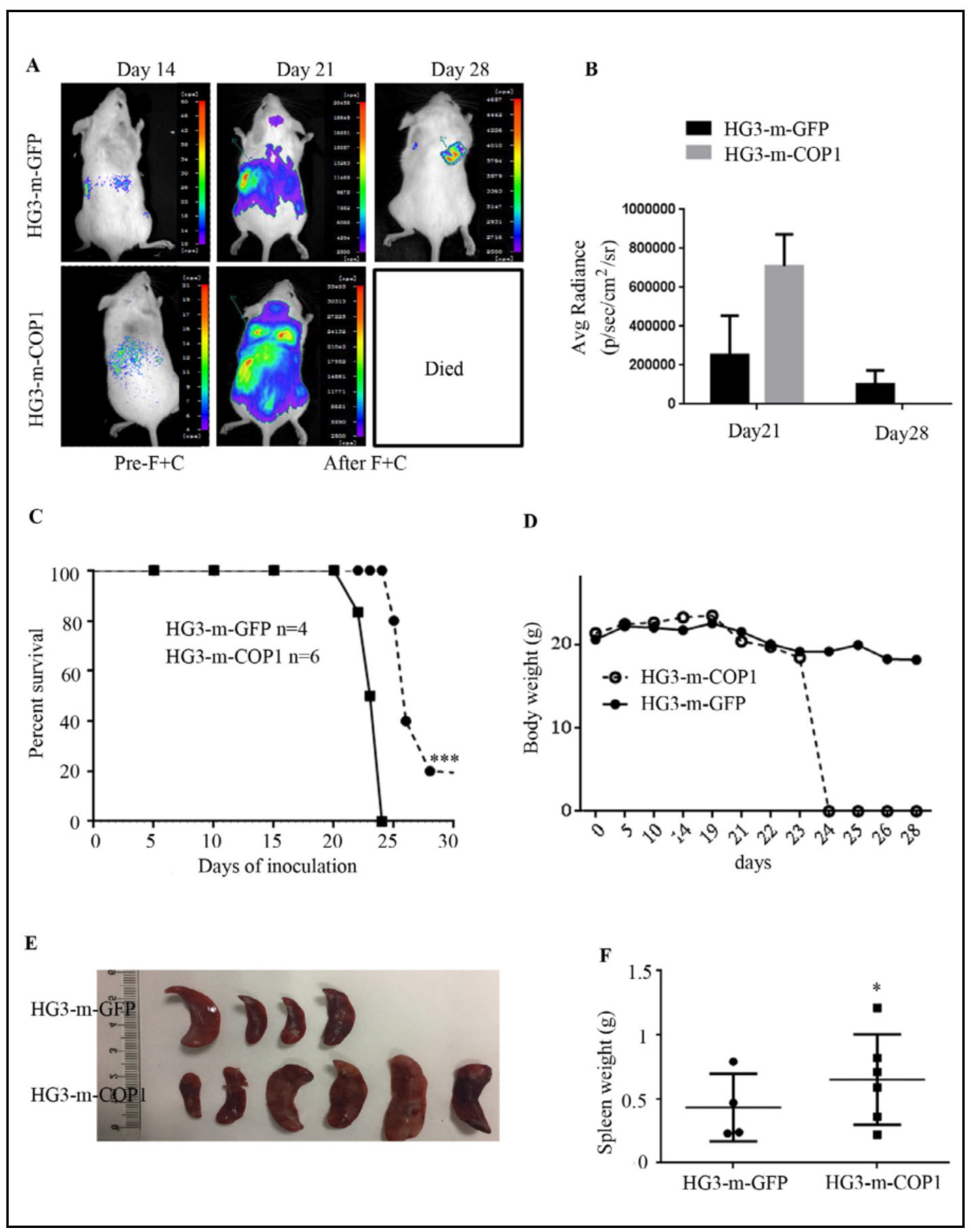

Fig. 5. HG3-m-COP1 transplanted mice shows short survival after $\mathrm{F}+\mathrm{C}$ therapy. (A) in vivo luciferase assay of mice transplanted with $1 \times 10^{5}$ HG3-m-GFP or HG3-m-COP1. 14 days after transplant, mice were treated with $\mathrm{F}+\mathrm{C}$ daily and luciferase density detected at 21 and 28 days of $\mathrm{F}+\mathrm{C}$ treatment. (B) Quantification of luciferase signal obtained from luciferase-labeled mice. (C) Kaplan-Meier survival curves of HG3-m-GFP and HG3-mCOP1 transplanted mice. (D) body weight variation of HG3-m-GFP and HG3-m-COP1 transplanted mice. (E and F) Representative image of spleen size of HG3-m-GFP and HG3-m-COP1 mice and quantification of spleen weight for the two groups. 


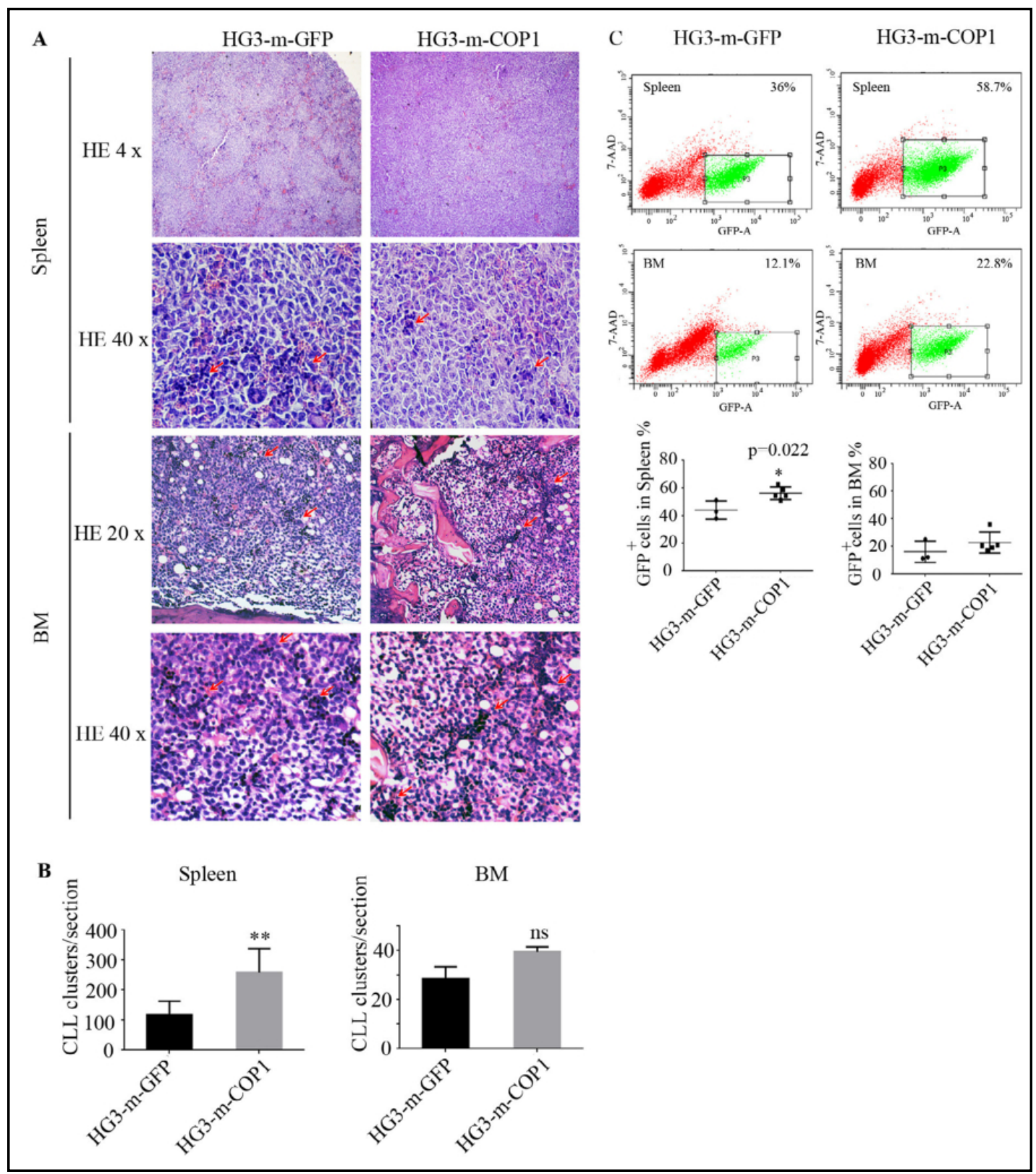

Fig. 6. BM and spleen features showing the evolution of the disease for HG3-m-GFP and HG3-m-COP1 mice. (A) H\&E staining of BM and spleen of HG3-m-GFP and HG3-m-COP1 transplant mice. (B) Quantification of infiltrating CLL cell clusters in spleen and BM of HG3-m-GFP and HG3-m-COP1 transplant mice. (C) The occupation of CLL cells in spleen or BM was detected by flow cytometry.

HG3-m-COP1 transplant mice showed low cell apoptosis rate when received $F+C$ therapy

To explain why the luciferase density was reduced in HG3-m-GFP transplant mice but not in HG3-m-COP1 mice after F+C therapy, we stained sections of the spleen and BM with cleaved-caspase 3 antibody to detect cell apoptosis. As expected, most of the CLL cells were negative staining for cleaved-caspase 3 in HG3-m-COP1 transplanted mice compared to HG3-m-GFP mice $\left({ }^{*} \mathrm{P}<0.05\right)$ (Fig. 7A and B). Moreover, The FACS results also showed that CLL cells with high COP1 expression presented low cell apoptosis ratio about $12 \%$ and $9.5 \%$ in spleen or BM, which is significantly lower than control CLL cells with $24 \%$ and $17.5 \%$ of apoptosis ratio in spleen or BM (7C). These data suggest that COP1 overexpression confers apoptosis resistance in vivo under a fludarabine treatment regimen. 


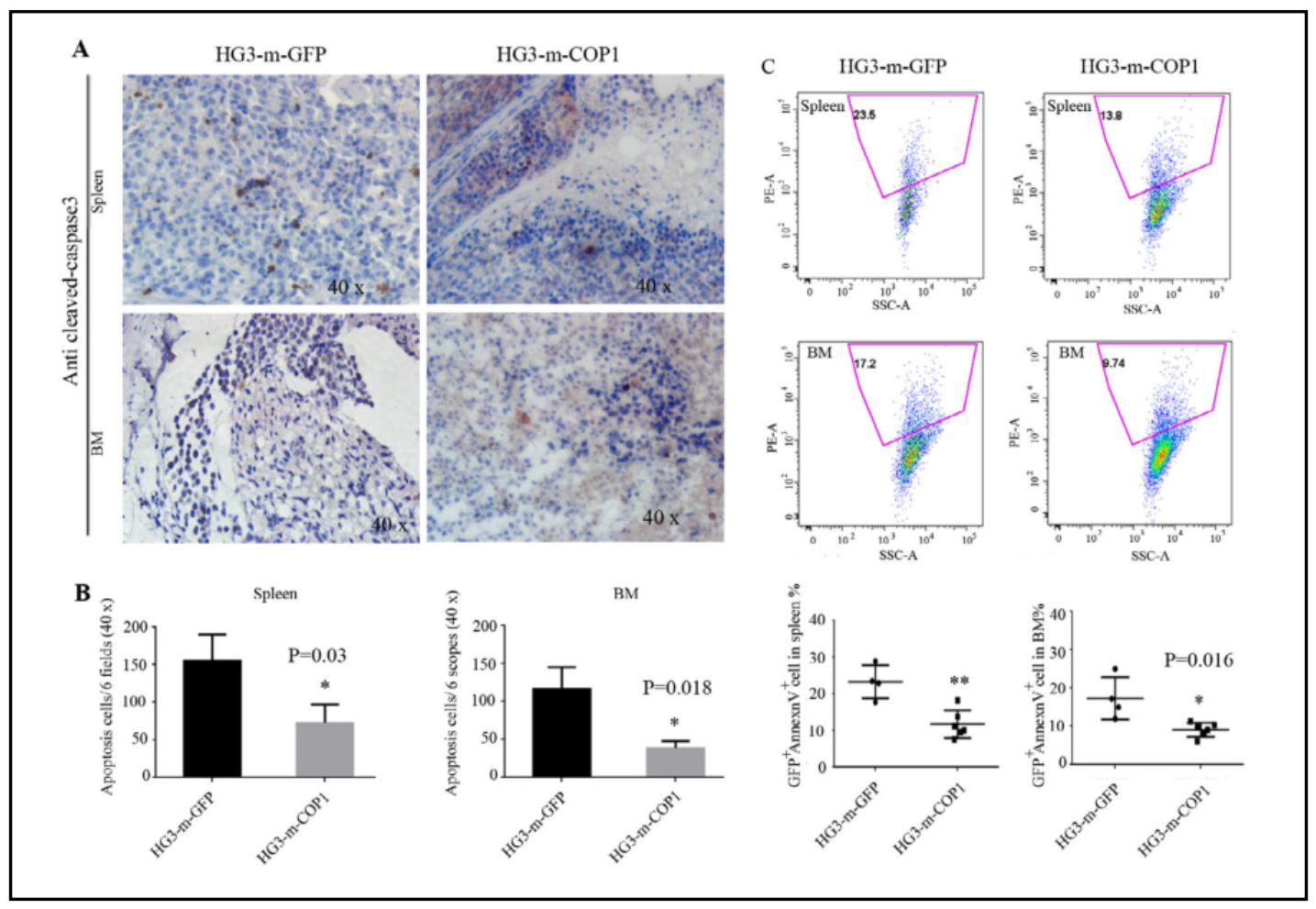

Fig. 7. COP1 overexpression diminished apoptosis of HG3 cells in vivo. (A) Immunohistochemistry of cleaved caspase-3 in BM and spleen of HG3-m-GFP and HG3-m-COP1 transplant mice. (B) Quantification of apoptotic cells in BM and spleen over 6 high power fields (HPF). (C) The apoptosis percentage of CLL cells in spleen and BM was detected by flow cytometry.

\section{Discussion}

CLL is the most common human leukemia, representing 30\% of all cases [25]. Despite CLL progression being slow and having a relatively long survival time, some patients have no obvious clinical presentation, the other part of patients presents lymphadenopathy, splenomegaly and anemia, and requiring treatment [26]. So far, the combination of chemotherapy with monoclonal antibodies has further improved response rates, while most of them have drug resistance [27]. Since the first-generation bruton's tyrosine kinase (BTK) inhibitor ibrutinib applies in CLL treatment, which effectively relieved resistant patients to the chemotherapy, but there is small portion of patients also show resistant because of BTK mutation at binding site of ibtutinib [28], while the second-generation is in clinical testing [29]. Thus, understanding of resistant mechanisms is important to design new target drug. In our previous study, we found that COP1 is up regulated in Binet C-phase CLL patients [8]. Here, we further showed COP1 may be an important factor to confer to drug resistance.

COP1, a E3 ubiquitin ligase, regulates ubiquitin-mediated degradation of proteins with opposing functions, including the tumor suppressor p53 and the oncoprotein c-Jun, thus creating a conundrum with respect to its role in cell proliferation and apoptosis [30]. We first found that COP1 overexpression in HG3 cells inhibited p53 accumulation induced by fludarabine. However, we didn't detect c-Jun expression. In subsequent ubiquitination experiments, we verified that COP1 was directly interacting with p53 and promoted ubitquitin dependent p53 degradation in HG3 cells.

In our previous study, we also demonstrated that COP1 overexpression inhibited p21 expression and increased HG3 cells proliferation and tumorigenicity [8]. Here, the downregulation of p53 by COP1 overexpression in HG3 cells disrupted the p53-Brn-3a complex. As reported, there is a common binding site for both p53 and Brn-3a located in the 


\section{Cellular Physiology Cell Physiol Biochem 2018;50:2314-2328

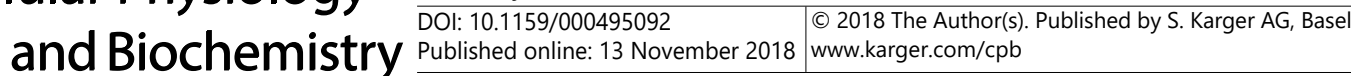 \\ Fu et al.: Constitutively Photomorphogenic 1 Reduces the Sensitivity of Chronic \\ Lymphocytic Leukemia Cells to Fludarabine}

Bcl-2 promoter. Brn-3a binding alone will promote Bcl-2 transcription, but once it complexes with p53, it binds less well and causes reduced Bcl-2 transcription [17]. We further present evidence that apoptosis resistance of HG3 cells in presence of fludarabine is caused by COP1mediated disruption of the p53-Brn-3a complex, leading to a sustained expression of the anti-apoptotic protein Bcl-2.

Animal models, in particular tumor cell transplant models and knockout mice, are an invaluable tool in CLL research [31-34]. Thus, we explored the ability of NPG mice, which lack $B$, T, and NK cells, to support the growth of the HG3 CLL cell line. We generated HG3-m-GFP and HG3-m-COP1 transplant mice 2 weeks after injection of the two cell lines. Interestingly, the two-week $\mathrm{F}+\mathrm{C}$ therapy improved survival of HG3-m-GFP transplanted mice, and reduced the tumor cells engraftment in spleen; this was not seen in HG3-m-COP1 engrafted mice. This was consistent with treatment of subcutaneous MEC-1 CLL cell line xenograft mice with $\mathrm{F}+\mathrm{C}$ therapy, which reduced the tumor growth and extended mice survival. Regrettably, we didn't observe long survival in all of HG3-m-GFP mice, perhaps because of the aggressiveness of the tumor or low effective $\mathrm{F}+\mathrm{C}$ therapy. In this study, we concluded that the reduced effect of $\mathrm{F}+\mathrm{C}$ therapy in HG3-m-COP1 mice is due to increased resistance to apoptosis of HG3 cells overexpressing COP1.

We also constructed HG3-m-GFP and HG3-m-shCOP1 transplanted mice to assess if loss of COP1 would delay the disease or extend mice survival. However, we failed to observe any difference in spleen weight (data not show), tumor cell engraftment or survival time (data not shown). This may be because p53 is not the only substrate for COP1 and other E3 ubiquitin ligases like MDM2 might play a compensatory role, but we didn't detect the MDM2 level in CLL patients or cell lines [35, 36].

\section{Conclusion}

In summary, COP1 maintains a normal cell survival and apoptosis by regulating the p53-Brn-3a-Bcl-2 signaling axis in normal cells, while COP1 overexpression disrupted the formation of p53-Brn-3a complex and promoted ubiquitin-mediated p53 degradation in HG3 cells, and increased apoptosis resistance of HG3 under fludarabine treatment. Moreover, COP1 overexpressing HG3 transplant mice showed a shorter survival even when $\mathrm{F}+\mathrm{C}$ therapy was administered. This study demonstrates that COP1 contributes to drug resistance of CLL cells to the fludarabine treatment in vitro and in vivo.

\section{Acknowledgements}

We are grateful to Dr Anders Rosén (Linköping University, Sweden) for generously providing HG3 cells. CF, XS, YG performed the experiments, and analyze the data. ZS and YW run all samples by flow cytometry. ZW, YY did mouse image. KX designed and wrote the paper. Christian Marinaccio and John. D Crispino reviewed the paper.

The present study was supported by the National Natural Science Foundation of China (81400127), and the Certificate of China Postdoctoral Science Foundation Grant (2015M571818), and six talent peaks project in Jiangsu Province (2016-WSN-134).

All animals' experiments was reviewed and approved by the Ethics Committee of Xuzhou Medical University.

\section{Disclosure Statement}

The authors have nothing to disclose. 


\section{Cellular Physiology Cell Physiol Biochem 2018;50:2314-2328 \begin{tabular}{l|l|l} 
DOI: 10.1159/000495092 & 2018 The Author(s). Published by S. Karger AG, Basel
\end{tabular} and Biochemistry Published online: 13 November 2018 www.karger.com/cpb}

Fu et al.: Constitutively Photomorphogenic 1 Reduces the Sensitivity of Chronic

Lymphocytic Leukemia Cells to Fludarabine

\section{References}

1 Malek SN: The biology and clinical significance of acquired genomic copy number aberrations and recurrent gene mutations in chronic lymphocytic leukemia. Oncogene 2013;32:2805-2817.

-2 Zhu DX, Zhu W, Fang C, Fan L, Zou ZJ, Wang YH, Liu P, Hong M, Miao KR, Liu P, Xu W, Li JY: miR-181a/b significantly enhances drug sensitivity in chronic lymphocytic leukemia cells via targeting multiple antiapoptosis genes. Carcinogenesis 2012;33:1294-1301.

-3 Austen B, Powell JE, Alvi A, Edwards I, Hooper L, Starczynski J, Taylor AM, Fegan C, Moss P, Stankovic T: Mutations in the ATM gene lead to impaired overall and treatment-free survival that is independent of IGVH mutation status in patients with B-CLL. Blood 2005;106:3175-3182.

-4 Rossi D, Cerri M, Deambrogi C, Sozzi E, Cresta S, Rasi S, De Paoli L, Spina V, Gattei V, Capello D, Forconi F, Lauria F, Gaidano G: The prognostic value of TP53 mutations in chronic lymphocytic leukemia is independent of Del17p13: implications for overall survival and chemorefractoriness. Clinical cancer research : an official journal of the American Association for Cancer Research 2009;15:995-1004.

5 Wickremasinghe RG, Prentice AG, Steele AJ: p53 and Notch signaling in chronic lymphocytic leukemia: clues to identifying novel therapeutic strategies. Leukemia 2011;25:1400-1407.

6 Marschitz I, Tinhofer I, Hittmair A, Egle A, Kos M, Greil R: Analysis of Bcl-2 protein expression in chronic lymphocytic leukemia. A comparison of three semiquantitation techniques. Am J Clin Pathol 2000;113:219229.

7 Podhorecka M, Halicka D, Klimek P, Kowal M, Chocholska S, Dmoszynska A: Resveratrol increases rate of apoptosis caused by purine analogues in malignant lymphocytes of chronic lymphocytic leukemia. Ann Hematol 2011;90:173-183.

-8 Fu C, Gong Y, Shi X, Shi H, Wan Y, Wu Q, Xu K: Expression and regulation of COP1 in chronic lymphocytic leukemia cells for promotion of cell proliferation and tumorigenicity. Oncology reports 2016;35:14931500 .

-9 Schwechheimer C, Deng XW: The COP/DET/FUS proteins-regulators of eukaryotic growth and development. Semin Cell Dev Biol 2000;11:495-503.

-10 Yi C, Wang H, Wei N, Deng XW: An initial biochemical and cell biological characterization of the mammalian homologue of a central plant developmental switch, COP1. BMC Cell Biol 2002;3:30.

11 Yi C, Deng XW: COP1 - from plant photomorphogenesis to mammalian tumorigenesis. Trends Cell Biol 2005;15:618-625.

12 Marine JC: Spotlight on the role of COP1 in tumorigenesis. Nature reviews. Cancer 2012;12:455-464.

13 Dornan D, Wertz I, Shimizu H, Arnott D, Frantz GD, Dowd P, O’Rourke K, Koeppen H, Dixit VM: The ubiquitin ligase COP1 is a critical negative regulator of p53. Nature 2004;429:86-92.

14 Dornan D, Shimizu H, Mah A, Dudhela T, Eby M, O’Rourke K, Seshagiri S, Dixit VM: ATM engages autodegradation of the E3 ubiquitin ligase COP1 after DNA damage. Science 2006;313:1122-1126.

15 Su CH, Zhao R, Velazquez-Torres G, Chen J, Gully C, Yeung SC, Lee MH: Nuclear export regulation of COP1 by 14-3-3sigma in response to DNA damage. Molecular cancer 2010;9:243.

16 Bunz F, Dutriaux A, Lengauer C, Waldman T, Zhou S, Brown JP, Sedivy JM, Kinzler KW, Vogelstein B: Requirement for p53 and p21 to sustain G2 arrest after DNA damage. Science 1998;282:1497-1501.

$\checkmark 17$ Hudson CD, Morris PJ, Latchman DS, Budhram-Mahadeo VS: Brn-3a transcription factor blocks p53mediated activation of proapoptotic target genes Noxa and Bax in vitro and in vivo to determine cell fate. The Journal of biological chemistry 2016;291:15909.

18 Dornan D, Bheddah S, Newton K, Ince W, Frantz GD, Dowd P, Koeppen H, Dixit VM, French DM: COP1, the negative regulator of $\mathrm{p} 53$, is overexpressed in breast and ovarian adenocarcinomas. Cancer research 2004;64:7226-7230.

19 Lee YH, Andersen JB, Song HT, Judge AD, Seo D, Ishikawa T, Marquardt JU, Kitade M, Durkin ME, Raggi C, Woo HG, Conner EA, Avital I, Maclachlan I, Factor VM, Thorgeirsson SS: Definition of ubiquitination modulator COP1 as a novel therapeutic target in human hepatocellular carcinoma. Cancer research 2010;70:8264-8269.

20 Li YF, Wang DD, Zhao BW, Wang W, Huang CY, Chen YM, Zheng Y, Keshari RP, Xia JC, Zhou ZW: High level of COP1 expression is associated with poor prognosis in primary gastric cancer. Int J Biol Sci 2012;8:11681177. 


\section{Cellular Physiology Cell Physiol Biochem 2018;50:2314-2328 \begin{tabular}{ll|l} 
and Biochemistry & $\overline{\text { DOl: 10.1159/000495092 }}$ & $\begin{array}{l}\text { C) } 2018 \text { The Author(s). Published by S. Karger AG, Basel } \\
\text { www.karger.com/cpb }\end{array}$ \\
\hline
\end{tabular}}

Fu et al.: Constitutively Photomorphogenic 1 Reduces the Sensitivity of Chronic Lymphocytic Leukemia Cells to Fludarabine

21 Yoshida A, Kato JY, Nakamae I, Yoneda-Kato N: COP1 targets C/EBPalpha for degradation and induces acute myeloid leukemia via Trib1. Blood 2013;122:1750-1760.

-22 Migliorini D, Bogaerts S, Defever D, Vyas R, Denecker G, Radaelli E, Zwolinska A, Depaepe V, Hochepied T, Skarnes WC, Marine JC: Cop1 constitutively regulates c-Jun protein stability and functions as a tumor suppressor in mice. The Journal of clinical investigation 2011;121:1329-1343.

-23 Lanemo Myhrinder A, Hellqvist E, Bergh AC, Jansson M, Nilsson K, Hultman P, Jonasson J, Buhl AM, Bredo Pedersen L, Jurlander J, Klein E, Weit N, Herling M, Rosenquist R, Rosen A: Molecular characterization of neoplastic and normal "sister" lymphoblastoid B-cell lines from chronic lymphocytic leukemia. Leuk Lymphoma 2013;54:1769-1779.

-24 Budhram-Mahadeo V, Morris PJ, Smith MD, Midgley CA, Boxer LM, Latchman DS: p53 suppresses the activation of the Bcl-2 promoter by the Brn-3a POU family transcription factor. The Journal of biological chemistry 1999;274:15237-15244.

-25 Pekarsky Y, Zanesi N, Croce CM: Molecular basis of CLL. Semin Cancer Biol 2010;20:370-376.

26 Molica S, Giannarelli D, Mirabelli R, Levato L, Gentile M, Lentini M, Morabito F: Changes in the incidence, pattern of presentation and clinical outcome of early chronic lymphocytic leukemia patients using the 2008 International Workshop on CLL guidelines. Expert Rev Hematol 2014;7:691-695.

27 Lu K, Wang X: Therapeutic advancement of chronic lymphocytic leukemia. J Hematol Oncol 2012;5:55.

28 Woyach JA, Furman RR, Liu TM, Ozer HG, Zapatka M, Ruppert AS, Xue L, Li DH, Steggerda SM, Versele M, Dave SS, Zhang J, Yilmaz AS, Jaglowski SM, Blum KA, Lozanski A, Lozanski G, James DF, Barrientos JC, Lichter P, Stilgenbauer S, Buggy JJ, Chang BY, Johnson AJ, Byrd JC: Resistance mechanisms for the Bruton's tyrosine kinase inhibitor ibrutinib. The New England journal of medicine 2014;370:2286-2294.

$29 \mathrm{Wu}$ J, Liu C, Tsui ST, Liu D: Second-generation inhibitors of Bruton tyrosine kinase. J Hematol Oncol 2016;9:80.

-30 Wei W, Kaelin WG, Jr: Good COP1 or bad COP1? In vivo veritas. The Journal of clinical investigation 2011;121:1263-1265.

-31 Simonetti G, Bertilaccio MT, Ghia P, Klein U: Mouse models in the study of chronic lymphocytic leukemia pathogenesis and therapy. Blood 2014;124:1010-1019.

32 Bertilaccio MT, Scielzo C, Simonetti G, Ponzoni M, Apollonio B, Fazi C, Scarfo L, Rocchi M, Muzio M, Caligaris-Cappio F, Ghia P: A novel Rag2-/-gammac-/--xenograft model of human CLL. Blood 2010;115:1605-1609.

33 Loisel S, Ster KL, Quintin-Roue I, Pers JO, Bordron A, Youinou P, Berthou C: Establishment of a novel human B-CLL-like xenograft model in nude mouse. Leuk Res 2005;29:1347-1352.

34 Loisel S, Le Ster K, Meyer M, Berthou C, Youinou P, Kolb JP, Billard C: Therapeutic activity of two xanthones in a xenograft murine model of human chronic lymphocytic leukemia. J Hematol Oncol 2010;3:49.

-35 Wang L, He G, Zhang P, Wang X, Jiang M, Yu L: Interplay between MDM2, MDMX, Pirh2 and COP1: the negative regulators of p53. Molecular biology reports 2011;38:229-236.

-36 Corcoran CA, Huang Y, Sheikh MS: The p53 paddy wagon: COP1, Pirh2 and MDM2 are found resisting apoptosis and growth arrest. Cancer Biol Ther 2004;3:721-725. 